\title{
Huang Scattering from Frenkel Defects in Electron-Irradiated F.c.c. Metals
}

\author{
By P. Ehrhart, U. Schlagheck AND W. Schilling \\ Institut für Festkörperforschung der KFA Jülich, Postfach 365, 517 Jülich, Germany (BRD)
}

(Received 29 April 1974)

\begin{abstract}
The diffuse scattering of X-rays $\left(\mathrm{Cu} K \alpha_{1}\right)$ has been investigated close to different Bragg reflexions of $\mathrm{Al}$ [Ehrhart, P. \& Schilling, W. (1973). Phys. Rer. B8, 2604] and Cu after low-temperature electron irradiation (defect concentrations between 2 and $6 \times 10^{-4}$ ). Measurements were done in directions parallel and perpendicular to the scattering vector. Recent results on copper are: (1) The symmetry of the long-range part of the displacement field around an interstitial has weakly tetragonal or cubic symmetry. (2) From comparisons of the diffuse scattering intensities with measurements of the latticeparameter change the absolute concentration and strength of the defects were determined. A reasonable set of values is: The volume change $\Delta V$ per interstitial is 1.45 atomic volumes, and the volume change by the relaxation around a vacancy is -0.4 atomic volumes. (3) During thermal annealing, besides the recombination of Frenkel pairs the formation of defect clusters is observed. These clusters contain about 5 to 10 interstitials at the end of stage I, about 80 interstitials at the end of stage II and 400 interstitials at the end of stage III. They form dislocation loops on $\{111\}$ or $\{110\}$ planes. During stage III small vacancy clusters are formed in addition to the interstitial clusters. The results for the larger clusters can be compared with observations in the electron microscope.
\end{abstract}

J. Appl. Cryst. (1975). 8, 150

\section{X-ray Studies of Defect Clusters in Copper*}

\author{
By BennetT C. LaRson \\ Solid State Division, Oak Ridge National Laboratory, Oak Ridge, Tennessee 37830, U.S.A.
}

(Received 29 April 1974; accepted 3 September 1974)

\begin{abstract}
X-ray diffraction techniques provide powerful tools for the study of lattice defects in crystals. For the case of clustered defects, many of these techniques are based on the scattering properties of the strained regions surrounding the defects rather than on the scattering from the defects themselves. This is particularly true of Huang diffuse scattering, anomalous transmission, and lattice-parameter measurements; the interpretation of these measurements, therefore, relies on a detailed knowledge of the strains around the defects. The Fourier-transformed, long-range strain fields entering into the Huang diffuse scattering can be expressed in terms of the dipole force model, and in this work comparisons of calculations based on this model with measured scattering from spherical precipitates and dislocation loops in copper are reviewed. The use of integral diffuse scattering measurements, made with a two-axis diffractometer, are discussed in terms of a direct analysis of the defect systems in the form of defect concentrations and size distributions and this procedure is applied to the study of the effects of irradiation conditions and postirradiation annealing on the defect distributions in neutron-irradiated copper. The relation of these diffuse scattering measurements to lattice parameter, anomalous transmission, electron microscopy and electrical resistivity measurement is also discussed.
\end{abstract}

\section{Introduction}

Clustered defects have been the subject of a considerable number of $\mathrm{X}$-ray studies in recent years. These studies have made use of lattice-parameter change, decrease in anomalous X-ray transmission, and integral

* Research sponsored by the U.S. Atomic Energy Commission under contract with Union Carbide Corporation. and differential Huang diffuse scattering measurements. Such measurements carried out close to the Bragg reflections are most sensitive to the strain fields surrounding defect clusters and except for measurements made far out in the Brillouin zone, the scattering from the defect atoms themselves is small.

The theoretical framework for analyzing these measurements is largely contained in recent work by Dederichs $(1971,1973)$ and Trinkaus $(1972 a, 1972 b)$ and 